(1)

CrossMark

\title{
An Italian sacrifice to the COVID-19 epidemic
}

\author{
Stefano Nava ${ }^{1}$, Roberto Tonelli ${ }^{2,3}$ and Enrico M. Clini $\mathbb{1}^{3}$
}

Affiliations: 'University Hospital of Bologna S. Orsola-Malpighi, Respiratory Diseases and Respiratory Intensive Care Unit, Dept of Medical Sciences DIMES, University of Bologna Alma Mater, Bologna, Italy. ${ }^{2}$ Clinical and Experimental Medicine PhD Program, University of Modena Reggio Emilia, Modena, Italy. ${ }^{3}$ University Hospital of Modena Policlinico, Respiratory Diseases Unit, Dept of Medical and Surgical Sciences SMECHIMAI, University of Modena Reggio Emilia, Modena, Italy.

Correspondence: Enrico M. Clini, University of Modena, University Hospital of Modena Policlinico, Via del Pozzo 71, Modena, 41141, Italy. E-mail: enrico.clinidunimore.it

@ERSpublications

The report of Italian doctors who died during the COVID-19 outbreak should inform us not to forget https://bit.ly/3cbSbkU

Cite this article as: Nava S, Tonelli R, Clini EM. An Italian sacrifice to the COVID-19 epidemic. Eur Respir J 2020; 55: 2001445 [https://doi.org/10.1183/13993003.01445-2020].

As reported by Newsweek magazine on 4 April 2020 [1], coronavirus has killed over 100 doctors and nurses around the world, nearly half of whom are reported to be in Italy. This report, however, underestimated the size of the problem. To date, 151 doctors and more than 40 nurses have died in Italy from coronavirus disease 2019 (COVID-19).

Overall, infection rates among healthcare workers in Italy and Spain are reported to be $9 \%$ and $14 \%$, respectively, while in China [2] and other part of the world this number was lower. There is no clear answer yet as to why Italian doctors have been so badly affected, but several factors may have played a role.

First, the outbreak was somehow underestimated by scientists and politicians since they interpreted the data coming from China in a way that confirms their a priori beliefs that the virus would not seriously hit Italy (i.e. confirmation bias).

The Centre for Disease Prevention and Control in Europe pointed out that the impact of COVID-19 depended on the single country's level of preparation and ability to implement rapid responses. Unfortunately, the time "advantage" that Italy had after the Chinese outbreak was lost.

The increase in cases after 21 February 2020 (first case reported in Codogno) was fast, and so the human and technical resources to fight the virus were not guaranteed, leading to a more serious impact, i.e. a greater number of deaths among the most vulnerable sectors of society, especially medical professionals. Poor coordination by authorities and a slow reaction to take drastic and protective measures for the hospital personnel put those on the front line at risk. Most of the district and rural hospitals (where the first cases occurred) were completely unprepared to face the epidemic, thus they were immediately faced with a lack of masks, shields, glasses and protective suits. Compared with nurses, doctors were not trained and sufficiently skilled in both wearing and using this equipment [3].

Secondly, doctors were working under extreme pressure for many hours without any breaks or days off, probably decreasing their attention towards protection. This became even more dramatic when the first doctors got ill, thus "obliging" the colleagues on duty to work even harder to replace the reduced staff. 
Thirdly, critical care beds and negative pressure rooms ran out very quickly in the first few weeks of the outbreak, as hospitals were overloaded with patients with acute respiratory failure. Therefore, physicians rapidly implemented aerosol generating procedures in the general ward, such as oxygen via different routes of administration and respiratory devices (continuous positive airway pressure and bilevel positive airway pressure). To obviate the problem hospital staff usually chose rooms with natural ventilation (airflow of at least $160 \mathrm{~L} \cdot \mathrm{s}^{-1}$ per patient as suggested by the World Health Organization) but obviously this was a makeshift remedy.

In order to better understand the problem of "deaths in the field" and avoid speculations, we have performed a descriptive analysis of the colleagues who died. Data have been extracted from the National Federation of Medical Doctors (portale.fnomceo.it) which reported the daily bulletin of deaths. The present report is based on the available information as of 3 May 2020 (the latest update), just before re-opening (phase 2). A cross-check of each name/surname was made through a web search across local press and the individual doctors professional association to obtain any other useful information. We included only those who were active or called back from retirement (122 out of 154).

Table 1 shows the demographics of this population, while figure 1 shows the distribution of both the specialty and site of residence and activity of this population. In addition, the trend of deaths over the period compared with the last updated national epidemic outbreak [4] is also shown.

The geographical distribution of doctors' residence closely paralleled the distribution of the patient clusters, so we can rule out the fact that Italian hospitals and medical services run autonomously by different regions may have created some inequalities.

The median age of the deceased colleagues was quite high, which might reflect the expected death rate in the patients with positive swabs in the general population ( $>10 \%$ in the age range $60-70$ years). However, we cannot exclude the role of their lower performance (i.e. in adapting to the hospital transformation in response to the emergency, the ability to put on protective equipment, etc.) as reported in older versus younger physicians [5].

Finally, but probably most importantly, there was the impact of the specialty of the doctors who died. The large majority of them worked as general practitioners (GPs) in or close to the area badly hit by the virus (i.e. Bergamo in Lombardy). The hospitals in that province were filled very quickly with patients. The authorities told people to only go to the Emergency Room when their temperature was $>37.5 \mathrm{C}$, even if prolonged over days and associated with dyspnoea. GPs in Italy also had the responsibility of going to the patient's house when needed (i.e. the patient cannot go to the doctor's office because she/he is too ill). GPs were the last to receive personal protective equipment, and very often had only a few surgical masks which had to be reused for days. Therefore, it is very likely that both in their office and at the patients home they visited many patients without the desirable protection.

The second most represented category were dentists, the large majority of them also had a degree in medicine. They had to buy the personal protective equipment themselves as they are not employees of the National Health Care System, but unfortunately pharmacies ran out of mask and shields very quickly, due to a huge demand from all the citizens. Thus, they operated for several days/weeks very close to the patient's airways with insufficient protection. Following this, during the outbreak, dentists were locked down by law (except for emergencies) so the number of dentists affected by COVID-19 dramatically decreased.

$\begin{array}{lr}\text { TABLE } 1 \text { Demographics of Italian doctors who died during the COVID-19 epidemic } \\ \text { Male } & 115(94.3) \\ \text { Age years } & 68(63-71) \\ \text { Age class years } & 2(1.6) \\ <50 & 14(11.5) \\ 51-60 & 70(57.4) \\ 61-70 & 35(28.7) \\ 71-80 & 1(0.8) \\ 81-90 & \\ \text { Service status } & 114(93.4) \\ \text { Active } & 8(6.6) \\ \text { Back from retirement } & \end{array}$

Data are presented as $\mathrm{n}(\%)$ or median (interquartile range). 
a)

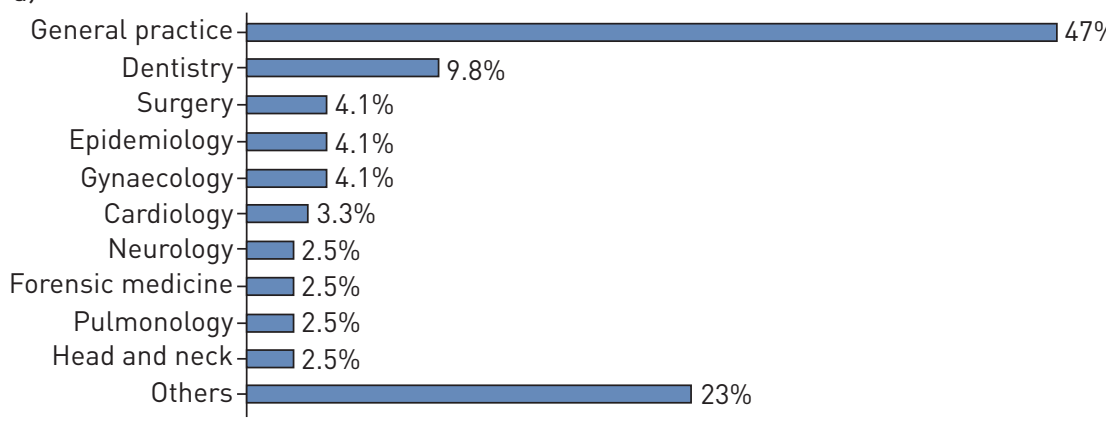

b)

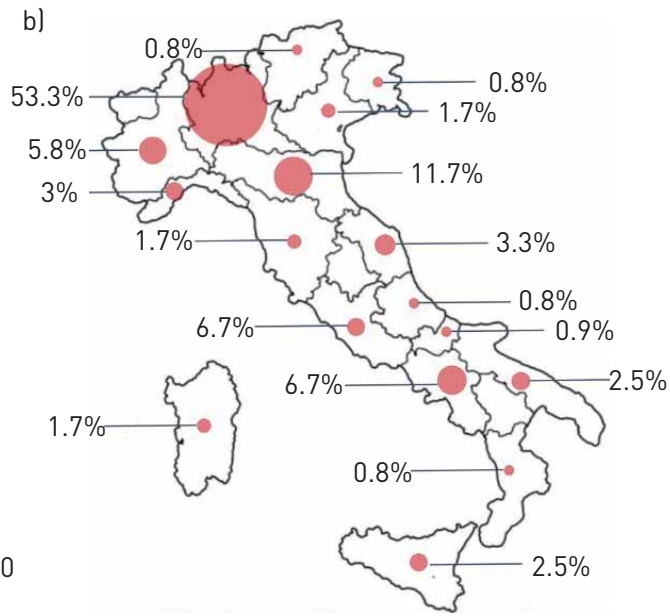

New positive cases per day

c) $7000_{7}$ Deaths among doctors per day

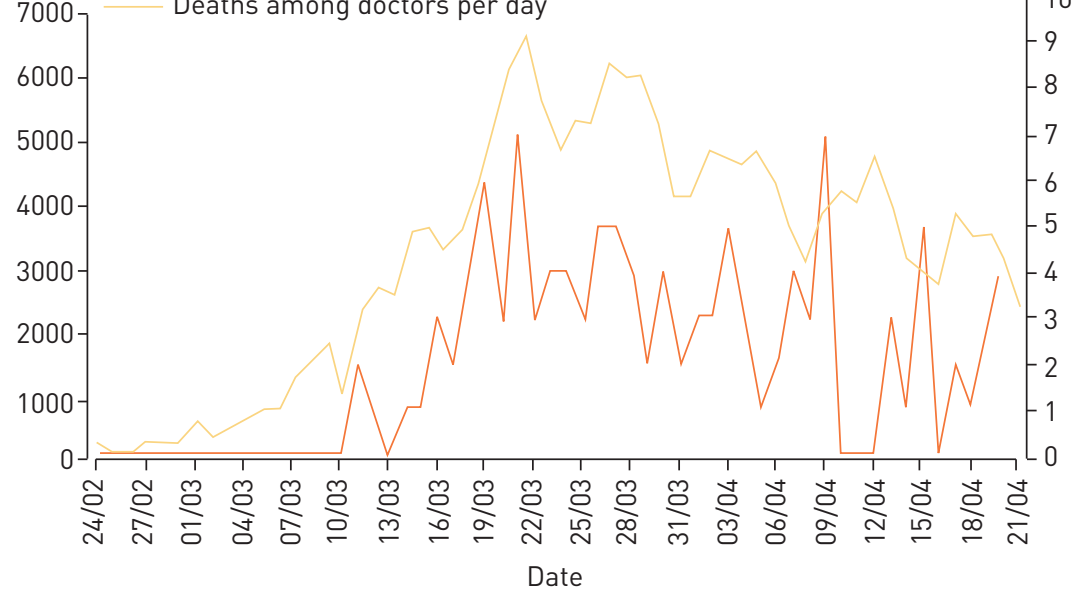

FIGURE 1 a) Specialties and b) geographical distribution of doctors who died during the COVID-19 epidemic in Italy. c) Time course and frequency of cases in the general population and deaths among doctors.

Quite surprisingly, doctors in critical care and emergency areas, as well pulmonologists, were spared by the infection, despite working on the frontline. This may simply mean that they are more used to working in their everyday practice with patients at risk for airway-derived infections, and therefore are probably more cautious and skilled in using and wearing the personal protective equipment.

In conclusion, and quite interestingly, the distribution of deaths among the medical doctors during the epidemic closely mirrors the curve of new cases among the Italian population. This confirms that the exposure to risk on the front line also reflects a similar inauspicious outcome of doctors on duty.

It is hard to face the sacrifice humans have payed in this dramatic COVID-19 epidemic, leaving us alone with thousands of thoughts and regrets, but it should also teach us not to forget.

Conflict of interest: None declared.

\section{References}

1 Newsweek. Over 100 doctors and nurses have died combating coronavirus across the world. www.newsweek.com/ coronavirus-deaths-infections-doctors-nurses-healthcare-workers-medical-staff-1496056. Date accessed 27 April 2020.

2 Xiang YT, Jin Y, Wang Y, et al. Tribute to health workers in China: a group of respectable population during the outbreak of the COVID-19. Int J Biol Sci 2020; 16: 1739-1740.

3 Li L, Xv Q, Yan J. COVID-19: the need for continuous medical education and training. Lancet Respir Med 2020; 8: e23.

4 Protezione Civile. Coronavirus emergency: the national answer. http://www.protezionecivile.gov.it/attivita-rischi/ rischio-sanitario/emergenze/coronavirus. Date last accessed 27 April 2020.

5 Choudhry NK, Fletcher RH, Soumerai SB. Systematic review: the relationship between clinical experience and quality of health care. Ann Intern Med 2005; 142: 260-273. 